\title{
Avaliação do Desempenho Reprodutivo de Novilhas de Corte Primíparas aos Dois Anos de Idade ${ }^{1}$
}

\author{
Marta Gomes da Rocha², José Fernando Piva Lobato ${ }^{3}$
}

\begin{abstract}
RESUMO - Objetivou-se com este trabalho avaliar o desempenho reprodutivo de novilhas de corte, primíparas aos dois anos de idade ('sistema um ano'). Essas novilhas foram submetidas a sistemas de alimentação diferenciados no primeiro outono/inverno pósdesmama, por 88 dias: a) pastejo contínuo em pastagem cultivada de azevém (L. multiflorum Lam) (PAST); b) pastejo contínuo em pastagem natural e suplementação energético-protéica (CNR); c) confinamento, a céu aberto, com silagem de sorgo + nitrogênio não protéico (SIL). Após esse período, as novilhas pastejaram em conjunto pastagens naturais melhoradas compostas por azevém, trevo branco (T. repens) e cornichão (L. corniculatus). Os dados foram coletados na Empresa Agropecuária Guatambu, Dom Pedrito, RS, em 395 bezerras de corte pertencentes a três grupos genéticos: Hereford (H); 3/4 Hereford-1/4 Nelore- (1/4 N); 5/8 Hereford-3/8 Nelore$(3 / 8 \mathrm{~N})$. Foram tomados registros de peso vivo (PV), condição corporal (CC), taxa de prenhez (Pr), parição (Par) e desmama (Desm). Novilhas alimentadas exclusivamente em pastagens tiveram maior PV e CC no final do período de acasalamento que novilhas do CNR e SIL, que não diferiram entre si. Sistemas alimentares não interferiram nas taxas de $\mathrm{Pr}$, Par e Desm. Novilhas $\mathrm{H}$ e $1 / 4 \mathrm{~N}$ foram mais produtivas no 'sistema um ano' ( 51,8 e 48,6 \% de Desm; $\mathrm{P}<0,01)$, superiores às novilhas $3 / 8 \mathrm{~N}(26,4 \%$ de Desm; $\mathrm{P}<0,01)$.
\end{abstract}

Palavras-chave: cruzamentos, idade ao primeiro parto, novilhas de corte, sistemas de produção

\section{Evaluation of Reproductive Performance of Primiparous Beef Heifers at Two Years Old}

\begin{abstract}
The experiment evaluated the reproductive performance of primiparous beef heifers at two years old ('one year system'). These heifers data were collected at Agropecuaria Guatambu, Dom Pedrito, RS, of 395 beef calves from three genetic groups, Hereford (H); 3/4 Hereford -1/4 Nellore (1/4 N); 5/8 Hereford- 3/8 Nellore (3/8 N). The heifers were submitted to three alternatives autumn-winter feeding systems a) continuous grazing on cultivated annual ryegrass (L. multiflorum Lam) (PAST); b) continuous grazing on natural pasture plus energy and protein supplementation (CNR); c) feedlot, with sorghum silage plus non proteic nitrogen (SIL). After 88 days, the animals of the three alternatives grazed together on improved natural pastures (L.multiflorum; T.repens; L.corniculatus). Liveweight (LW), body condition (BC), pregnancy rate (Pr), calving (C) and weaning rate (W) were recorded. At the end of mating period PAST heifers were heavier and had greater BC than SIL and CNR. The use of different feeding systems did not show significant differences in $\mathrm{Pr}, \mathrm{C}, \mathrm{W}$. H and 1/4 N primiparous cows were more productive at 'one year system' with greater weaning rates (51.8 and $48.6 \% ; \mathrm{P}>0.05)$ than $3 / 8 \mathrm{~N}(26.4 \% ; \mathrm{P}<0.01)$.
\end{abstract}

Key Words: age at first calving, beef heifers, crossbreeding, production systems

\section{Introdução}

O primeiro parto de novilhas de corte aos dois anos, conhecido como 'sistema um ano', decorre de um conjunto de práticas de alimentação e manejo dentro de um rebanho de cria associado a genética animal para puberdade precoce. No Rio Grande do Sul, acompanhando uma tendência mundial de intensificação da produção de bovinos de corte, existem empresas rurais onde novilhas são acasaladas pela primeira vez aos 14-15 meses de idade. Nesses locais, o ponto central de interesse no sistema 'um ano' está no conhecimento de que existe maior eficiência biológica nas fêmeas que parem pela primeira vez aos dois anos de idade. Nesse sistema haveria, potencialmente, maior lucratividade, pois a vaca produziria mais quilogramas de bezerros durante sua vida.

Quando a idade das novilhas colocadas em reprodução é superior a 14 meses, existe muita flexibilidade na quantidade e na época de aquisição do ganho de peso necessário entre a desmama e o início da estação de acasalamento. No entanto, para assegurar um desenvolvimento corporal adequado para o acasalamento das novilhas no 'sistema um ano', é necessário um nível alimentar alto e contínuo para o rebanho. As bezerras são tradicionalmente desma-

\footnotetext{
${ }_{1}^{1}$ Parte da tese de doutorado apresentada à UFRGS, pelo primeiro autor.

${ }^{2}$ Dra., Professor Adjunto II, do Departamento de Zootecnia da UFSM. E.mail: mrocha@creta.ccr.ufsm.br

${ }^{3} \mathrm{PhD}$., Professor Adjunto IV, do Departamento de Zootecnia da UFRGS. Bolsista IA, CNPq. Porto Alegre, Caixa Postal, 776, CEP 90.001-
} 970, Porto Alegre, RS. E.mail: lobato@orion.ufrgs.br 
madas no outono, sendo necessário assegurar ganho de peso satisfatório destes animais nos 100 dias posteriores à desmama, o que constitui um desafio no sistema que prevê novilhas primíparas aos dois anos de idade. A possível ocorrência de fatores climáticos adversos ao estabelecimento e manejo correto das pastagens de inverno pode prejudicar o acasalamento aos 14-15 meses de idade em situação exclusiva de pastejo. A utilização de forragem conservada ou ração comercial, nesse período, surge como alternativa para diminuir o risco de insucesso no 'sistema um ano'.

No Rio Grande do Sul e no Brasil, são escassos os dados disponíveis sobre acasalamento de novilhas aos 14-15 meses e seu comportamento reprodutivo quando primíparas aos 24 meses (Magalhães et al., 1990; Beretta \& Lobato, 1998; Pötter et al., 1998). Nos sistemas de produção de bovinos de corte, a importância da idade das vacas ao primeiro parto reside na redução do intervalo de gerações, na capacidade de afetar o progresso genético do rebanho, no peso e número de bezerros comercializáveis. A redução na idade de acasalamento de novilhas de corte também diminui a participação de animais improdutivos ou em recria na composição do rebanho.

Com o objetivo de contribuir para a determinação de alguns parâmetros regionais relativos ao sistema 'um ano' de produção de bovinos de corte, foram estudados neste trabalho as características de reprodução de novilhas de três grupos genéticos, primíparas aos dois anos de idade e submetidas a três sistemas alimentares distintos no período de inverno.

\section{Material e Métodos}

O experimento foi conduzido na Empresa Agropecuária Guatambu, município de Dom Pedrito, região da Campanha no Rio Grande do Sul. O clima, segundo a classificação de Köppen (Moreno, 1991), é do tipo Cfa (sub-tropical úmido). Foram utilizadas 395 bezerras de corte pertencentes a três grupos genéticos: Hereford $(\mathrm{H} ; \mathrm{n}=139) ; 3 / 4$ Hereford $-1 / 4$ Nelore- $(1 / 4 \mathrm{~N} ; \mathrm{n}=146)$ e $5 / 8$ Hereford- $3 / 8$ Nelore (3/ $8 \mathrm{~N} ; \mathrm{n}=110)$. Os animais foram avaliados do nascimento, na primavera de 1993, até a desmama de seus bezerros, no outono de 1996.

Após a desmama, em março de 1994, os animais permaneceram em pastagem natural melhorada com trevo branco e cornichão até $02 / 06$ quando, equilibrados por grupo racial, foram manejados por 88 dias em três tratamentos alimentares: a) pastejo contínuo em pastagem de azevém (L. multiflorum Lam.) - PAST; b) pastejo contínuo em pastagem natural e suplementação com ração - CNR; c) confinamento, a céu aberto, recebendo à vontade, silagem de sorgo + nitrogênio não proteico - SIL .

As novilhas do tratamento PAST $(\mathrm{n}=143)$ foram manejadas em pastagem de azevém (L. multiflorum Lam.), com disponibilidade média de $1100 \mathrm{~kg}$ de MS/ ha e carga animal média de $350 \mathrm{~kg}$ de peso vivo/ha. A disponibilidade de forragem foi medida através da técnica da dupla amostragem.

As novilhas do CNR $(n=119)$ permaneceram em pastagem natural e foram suplementadas com ração comercial (18\% de PB e $72 \%$ de NDT), na quantidade diária de 1,5\% em matéria seca (MS) do peso corporal dos animais, ajustada a cada 28 dias. A ração foi ofertada às 14 horas, diariamente. $O$ campo nativo foi roçado e permaneceu diferido por 45 dias antes da entrada dos animais no potreiro. Durante o período experimental a disponibilidade média de matéria seca da pastagem foi de $900 \mathrm{~kg}$ /ha e a carga animal média de $220 \mathrm{~kg}$ de peso vivo/ha.

Os animais do SIL $(n=133)$ receberam silagem de sorgo $(5,2 \%$ de $\mathrm{PB}$ e $61 \%$ de NDT) à vontade. A quantidade 'alvo' de nitrogênio não protéico/bezerra/ dia foi de $0,1 \mathrm{~kg}$, sendo uma mistura de uréia + sulfato de amônio, na proporção de 9:1. Os animais permaneceram em uma área de 10 ha. $\mathrm{O}$ alimento foi distribuído às 9 e às 15 horas.

Após a aplicação dos três sistemas alimentares as novilhas permaneceram como um só rebanho, em pastagem melhorada com azevém, trevo branco e cornichão até o final do período reprodutivo.

O período reprodutivo foi de 77 dias (10/11/ 1994 a 25/01/1995), sendo os primeiros 45 dias de inseminação artificial e os restantes 32 dias por monta natural. Esta foi realizada estando as novilhas em potreiros com composição botânica e disponibilidades semelhantes, mas separadas por grupo racial. Após o término da estação reprodutiva os animais permaneceram em sistema exclusivo de pasto. O diagnóstico de gestação foi feito por palpação retal, no período compreendido entre 06/03 a 08/03/1995.

Todos os animais tiveram acesso à mistura mineral à vontade e foram submetidos ao controle sanitário usual da propriedade.

As pesagens foram realizadas com jejum prévio de 12-14 horas. A cada pesagem, as fêmeas foram 
submetidas a uma avaliação subjetiva de sua condição corporal (CC) por dois avaliadores. Os critérios utilizados foram adaptados da classificação de Lowman et al. (1973), em que a condição varia de 1 (magra) a 5 (gorda).

Os dados de prenhez, parição e desmama foram comparados pelo teste de qui-quadrado (Steel \& Torrie, 1980). A análise estatística para as variáveis peso e condição corporal foi realizada usando o procedimento GLM do pacote computacional SAS (1989). O modelo linear incluiu grupo racial, sistemas alimentares no outono/inverno pós-desmama e categoria da vaca mãe da bezerra (grau de sangue e número de partos) dentro de grupo racial, grupo racial x sistema alimentar e categoria de vaca $\mathrm{x}$ sistema alimentar como variáveis independentes. Interações não significativas foram colocadas no erro experimental.

\section{Resultados e Discussão}

O ganho diário médio (GDM) entre 02/06/94 a 28/ 08/94, período de aplicação dos sistemas alimentares, e a CC em 28/08/94, final de aplicação dos sistemas, são apresentados na Tabela 1 . Os tratamentos no período pós-desmama não determinaram diferenças no $\operatorname{GMD}(\mathrm{P}>0,05)$. A CC inicial média foi de 3,1 para os três tratamentos. Animais alimentados com silagem apresentaram CC superior $(\mathrm{P}<0,01)$ no final da aplicação dos tratamentos. Esta propensão para estocar energia nas novilhas alimentadas com silagem pode ser importante no estabelecimento da CC necessária para prenhez aos 14-15 meses de idade.

Tabela 1- Média dos quadrados mínimos para efeito do sistema alimentar pós-desmame no ganho diário médio (GDM) e condição corporal (CC) durante o período de $02 / 06$ a $28 / 08$

Table 1 - Least square means for the effect of feeding system on live weight gain (LWG) and body condition (BC) during the period (06/02 to 08/28)

\begin{tabular}{lcc}
\hline $\begin{array}{l}\text { Sistema alimentar } \\
\text { Feeding system }\end{array}$ & $\begin{array}{c}\text { GDM, kg/dia } \\
L W G, k g / d a y\end{array}$ & $\begin{array}{c}\mathrm{CC}, 1-5 \\
B C, 1-5\end{array}$ \\
\hline $\begin{array}{l}\text { Pastagem natural } \\
\text { Natural pasture }\end{array}$ & 0,429 & $3,2^{\mathrm{b}}$ \\
$\begin{array}{l}\text { Silagem de sorgo }+\mathrm{NNP} \\
\text { Sorghum silage }+ \text { NNP }\end{array}$ & 0,412 & $3,3^{\mathrm{a}}$ \\
$\begin{array}{l}\text { Pastagem cultivada } \\
\text { Cultivated pasture }\end{array}$ & 0,390 & $3,2^{\mathrm{b}}$ \\
\hline
\end{tabular}

a, b - na mesma coluna, diferem significativamente $(P<0,01)$. $a, b$ - at same column, are statiscally different $(P<.01)$.

R. Bras. Zootec., v.31, n.3, p.1388-1395, 2002 (suplemento)
Bezerras que saíram dos tratamentos alimentares do outono/inverno em condições similares de peso $(\mathrm{P}>0,05)$, com média de $177 \mathrm{~kg}$, realizaram ganhos distintos $(\mathrm{P}<0,01)$ no período logo após o término da aplicação dos tratamentos e antes do início da estação reprodutiva. No período de 28/08 a 06/10, bezerras oriundas do CNR, SIL e PAST foram colocadas em um só lote em campo nativo melhorado e realizaram GDM de 0,254, 0,311 e 0,695 kg/dia, respectivamente. No período de $06 / 10$ a 09/11, média de todos os tratamentos, o GMD foi de $0,954 \mathrm{~kg}$. O ganho diferenciado e maior $(\mathrm{P}<0,01)$ para as novilhas do PAST, no período de $28 / 08$ a $06 / 10$ determinou, no início da estação de acasalamento, que as novilhas tivessem seu peso vivo afetado $(\mathrm{P}<0,01)$ pelo sistema de alimentação anterior, na fase pós-desmame (Tabela 2).

Novilhas alimentando-se somente de pastagens desde a desmama foram mais pesadas em 10/11, início do acasalamento, e em 25/01, final do acasalamento, do que suas contemporâneas alimentadas na pós-desmama no sistema CNR ou SIL que não diferiram entre si $(\mathrm{P}>0,05)$. Novilhas da SIL realizaram maior GMD durante a estação de acasalamento $(\mathrm{P}<0,01)$ do que novilhas do $\mathrm{CNR}$ e da PAST, que diferiram entre si $(\mathrm{P}<0,01)$. Esses GDM diferenciados não foram suficientes, no entanto, para compensar o peso desses animais por ocasião do início do acasalamento.

Grande número de estudos mostrou uma relação positiva entre peso vivo no primeiro acasalamento e taxa de natalidade subsequente em novilhas Angus e Hereford (Carter \& Cox, 1973; Ellis, 1974; Milagres et al., 1979) e em outras raças (Sawyer et al., 1991a). A economicidade do peso, no início do acasalamento, dentro de cada tratamento, depende do valor marginal dos bezerros obtidos e alimentos, os quais podem ser suplementos caros quando a disponibilidade e qualidade das pastagens é limitante.

Observa-se na Tabela 2 que o grupo racial afetou de forma significativa o peso no início da estação de acasalamento $(\mathrm{P}<0,05)$. Novilhas $\mathrm{H}$ apresentaram maior peso $(\mathrm{P}<0,01)$ do que as novilhas cruzas, que não diferiram entre si $(\mathrm{P}>0,05)$. Nessa ocasião, as $\mathrm{H}$ foram 14,8 e $18,8 \mathrm{~kg}$ mais pesadas que as $1 / 4 \mathrm{Ne} 3 / 8 \mathrm{~N}$, respectivamente. Esses dados diferem das observações de Bayley et al. (1988), os quais mostram novilhas oriundas de cruzamentos com Bos indicus significativamente mais pesadas no início do acasalamento que novilhas Hereford. No final do 
Tabela 2 - Média dos quadrados mínimos para efeito do sistema alimentar pós-desmame e grupo racial no peso vivo (PV), condição corporal (CC) e ganho diário médio (GDM) durante o acasalamento

Table 2 - Least square means for the effects of feeding system and genetic group on live weight (LW), body condition (BC) and daily live weight gain (LWG) at mating period

\begin{tabular}{|c|c|c|c|c|c|}
\hline \multirow[t]{2}{*}{$\begin{array}{l}\text { Sistema alimentar } \\
\text { Feeding system }\end{array}$} & \multicolumn{2}{|c|}{$\begin{array}{l}\mathrm{PV}, \mathrm{kg} \\
L W, k g\end{array}$} & \multicolumn{2}{|c|}{$\begin{array}{l}C C, 1-5 \\
B C, 1-5\end{array}$} & \multirow[t]{2}{*}{$\begin{array}{l}\mathrm{GDM}, \mathrm{kg} / \mathrm{dia} \\
L W G, \mathrm{~kg} / \text { day }\end{array}$} \\
\hline & $\begin{array}{l}\text { IA } \\
B M\end{array}$ & $\begin{array}{l}\text { FA } \\
E M\end{array}$ & $\begin{array}{l}\text { IA } \\
B M\end{array}$ & $\begin{array}{l}\text { FA } \\
E M\end{array}$ & \\
\hline $\begin{array}{l}\text { Pastagem natural } \\
\text { Natural pasture }\end{array}$ & $236,41^{b}$ & $273,61^{b}$ & $3,40^{\mathrm{c}}$ & $3,68^{b}$ & $0,496^{\mathrm{b}}$ \\
\hline $\begin{array}{l}\text { Silagem de sorgo }+\mathrm{NNP} \\
\text { Sorghum silage }+N N P\end{array}$ & $235,88^{b}$ & $275,55^{b}$ & $3,50^{\mathrm{b}}$ & $3,74^{\mathrm{b}}$ & $0,529^{\mathrm{a}}$ \\
\hline $\begin{array}{l}\text { Pastagem cultivada } \\
\text { Cultivated pasture } \\
\text { Grupo genético }\end{array}$ & $248,34^{\mathrm{a}}$ & $283,21^{\mathrm{a}}$ & $3,60^{\mathrm{a}}$ & $3,81^{\mathrm{a}}$ & $0,465^{\mathrm{c}}$ \\
\hline $\begin{array}{l}\text { Genetic group } \\
\text { Hereford } \\
\text { Hereford }\end{array}$ & $251,59^{\mathrm{A}}$ & $278,41^{\mathrm{AB}}$ & $3,61^{\mathrm{A}}$ & $3,78^{\mathrm{A}}$ & $0,358^{\mathrm{C}}$ \\
\hline $\begin{array}{l}\text { 3/4 Hereford-1/4 Nelore- } \\
3 / 4 \text { Hereford-1/4 Nellore/ }(1 / 4 \mathrm{~N})\end{array}$ & $236,78^{\mathrm{B}}$ & $280,58^{\mathrm{A}}$ & $3,55^{\mathrm{AB}}$ & $3,81^{\mathrm{A}}$ & $0,584^{\mathrm{A}}$ \\
\hline $\begin{array}{l}5 / 8 \text { Hereford- } 3 / 8 \text { Nelore } \\
5 / 8 \text { Hereford-3/8 Nellore }(3 / 8 N)\end{array}$ & $232,77^{\mathrm{B}}$ & $274,02^{\mathrm{B}}$ & $3,48^{\mathrm{B}}$ & $3,64^{\mathrm{B}}$ & $0,550^{\mathrm{B}}$ \\
\hline
\end{tabular}

IA: Início do acasalamento (10/11) (BM: Beginning of mating period [11/10]).

FA: Final do período de acasalamento (25/01) (EM: End of mating period [01/25]).

a, b, c - na mesma coluna, diferem significativamente $(P<0,05)(a, b, c-$ at same column, are statiscally different $[P<.05])$.

acasalamento as novilhas $3 / 8 \mathrm{~N}$ continuaram mais leves, mas sem diferir das $\mathrm{H}(\mathrm{P}>0,05)$ e novilhas $1 / 4$ $\mathrm{N}$ passaram a ser as mais pesadas, também sem diferir das $\mathrm{H}(\mathrm{P}>0,05)$. A maior adaptação dos animais cruzas Bos indicus $x$ Bos taurus às maiores temperaturas de final de primavera e início de verão, possivelmente foram determinantes do seu melhor desempenho durante a estação de acasalamento (Vercoe \& Frisch, 1982).

Na Tabela 2 também é observado que as novilhas apresentaram, entre o início e final do acasalamento, mudanças positivas na CC, mas de baixo valor. A CC mais baixa $(\mathrm{P}<0,01)$ no grupo $3 / 8 \mathrm{NH}$ sugere que este grupo teve menor oportunidade para atender suas exigências durante a estação de acasalamento. Essa $\mathrm{CC}$ de 3,64, no entanto, foi superior a CC de 2,8 em novilhas de mesma idade, no início do acasalamento, em dezembro, relatada por Beretta \& Lobato (1998), com animais de menor padrão zootécnico.

Observa-se na Tabela 3 que tratamentos alimentares na pós-desmama determinaram desempenho reprodutivo subsequente semelhante $(\mathrm{P}>0,05)$. Resultados similares a esse, também para 'sistema um ano', foram obtidos por Beretta \& Lobato (1998), os quais não observaram relação com o sistema de alimentação pós-desmama na proporção de novilhas ciclando em 07/12. Os autores ressaltam que o peso das novilhas no início do experimento teve influência significativa nos resultados, indicando que, além do sistema alimentar hibernal, o peso que as novilhas possuem ao início do inverno é determinante do peso com que elas chegam à época de serviço.

$\mathrm{O}$ peso dos animais do tratamento PAST foi superior $(\mathrm{P}<0,01)$ durante o acasalamento (Tabela 2$)$

Tabela 3 - Efeito dos sistemas alimentares no outono/ inverno pós-desmama sobre o percentual de prenhez, parição e desmama no 'sistema um ano'

Table 3 - Effect of post weaning feeding system on pregnancy rate, calving rate and weaning rate in 'one year' system

\begin{tabular}{lccc}
\hline $\begin{array}{l}\text { Sistema } \\
\text { System }\end{array}$ & $\begin{array}{c}\text { Prenhez } \\
\text { Pregnancy } \\
\%\end{array}$ & $\begin{array}{c}\text { Parição } \\
\text { Calving } \\
\%\end{array}$ & $\begin{array}{c}\text { Desmama } \\
\text { Weaning } \\
\%\end{array}$ \\
\hline $\begin{array}{l}\text { Pastagem natural } \\
\text { Natural pasture }\end{array}$ & 64,70 & 57,14 & 51,26 \\
$\begin{array}{l}\text { Silagem de sorgo + NNP } 52,63 \\
\begin{array}{l}\text { Sorghum silage + NNP } \\
\text { Pastagem cultivada }\end{array}\end{array}$ & 61,54 & 58,12 & 37,59 \\
$\begin{array}{l}\text { Cultivated pasture } \\
\text { Média }\end{array}$ & 59,39 & 51,78 & 42,66 \\
$\begin{array}{l}\text { Mean } \\
\text { Pean }\end{array}$ & & & 43,54 \\
\hline
\end{tabular}

$(P>0,05)(P>.05)$. 
em relação aos outros tratamentos mas, no entanto, não foi determinante de porcentuais de prenhez, parição ou desmama superiores $(\mathrm{P}>0,05)$. Também Reynolds et al. (1991) observaram que as taxas de prenhez não estiveram associadas com taxas de crescimento ou pesos atingidos em idades fixas.

A taxa média de parição observada no presente trabalho em primíparas aos 24 meses $(51,78 \%)$ foi semelhante a média de parição aos 48 meses no rebanho de corte do Rio Grande do Sul (Cachapuz, 1995). As taxas de prenhez obtidas não podem ser consideradas altas, o que constituiria a garantia da maior eficiência econômica e biológica do 'sistema um ano' com relação ao 'sistema dois anos' (Werth et al., 1991; McMillan et al., 1992). As taxas de prenhez alcançadas nos sistemas alimentares estão dentro da faixa relatada pela bibliografia para primíparas aos 24 meses (Short \& Bellows, 1971; McMillan e McCall, 1991; Reynolds et al., 1991; Núñez-Dominguez et al., 1991; Bergmann \& Hohenboken, 1992).

A diferença de $8,2 \%$ entre os índices de parto e desmama observada neste trabalho foi superior aos $5,25 \%$ relatados para o rebanho de corte da região da Campanha do Rio Grande do Sul (Mielitz Netto, 1994). Essa mortalidade até à desmama foi inferior aos 10,9 e $8,7 \%$ observados por Patterson et al. (1987) para primíparas com parto aos dois e três anos de idade, respectivamente.

Tendo sido observado desempenho reprodutivo similar em animais submetidos aos três sistemas alimentares na pós-desmama ( $\mathrm{P}>0,05)$, a escolha por uma destas técnicas será determinada pela relação entre preço produto/preço insumos determinando sua eficiência econômica, conforme discutido por Pötter et al. (1998).

Consta na Tabela 4 a porcentagem de prenhez, parição e desmama conforme o grupo racial. Novi- lhas Hereford e $1 / 4 \mathrm{~N}$ não diferiram entre si $(\mathrm{P}>0,05)$ e apresentaram taxas de prenhez, parição e desmama superiores ao grupo $3 / 8 \mathrm{~N}(\mathrm{P}<0,01)$. Esses dados, no entanto, diferem de vários trabalhos que relatam taxas de concepção semelhantes, em novilhas acasaladas aos 14 meses, nos grupos genéticos que atingem a puberdade em idade mais avançada e naqueles grupos que atingem a puberdade com menor idade (Young et al., 1978; Laster et al., 1979; Gregory et al., 1978). Patterson et al. (1991), no entanto, trabalhando com cruzas Bos taurus e Bos indicus, observaram maiores taxas de prenhez no grupo Bos taurus após 45 dias de acasalamento.

Em condições similares aos outros grupos raciais estudados, o resultado do grupo $3 / 8 \mathrm{~N}$, com 33,64 $\%$ de parição, evidenciou sua menor adaptação ao sistema de acasalamento aos 14-15 meses de idade. Nas novilhas $3 / 8 \mathrm{~N}$, maior tamanho e peso na maturidade em relação aos outros grupos estudados resultam em aumento das exigências nutricionais (NRC, 1996). Conforme Ferrel (1982), o desempenho reprodutivo em resposta a restrições alimentares tende a decrescer mais drasticamente em novilhas de maior tamanho adulto. Essas novilhas, com menor peso à desmama $(\mathrm{P}<0,01)$ que novilhas dos outros dois grupos genéticos (Rocha, 1997), necessitariam, certamente, de tratamento nutricional diferenciado para desempenho reprodutivo semelhante. No início do acasalamento (Tabela 2), o peso do grupo $3 / 8 \mathrm{~N}$ não diferiu do grupo 1/4 N ( $>>0,05)$, o qual apresentou desempenhos reprodutivos superiores. Isto pode ser considerado indicativo de que novilhas $3 / 8 \mathrm{NH}$ necessitariam de um peso mais elevado para melhor desempenho reprodutivo.

Conforme Sawyer et al. (1991b), em animais parindo aos dois anos de idade, as taxas e a época de

Tabela 4 - Efeito do grupo racial sobre o percentual de prenhez, parto e desmama no 'sistema um ano' Table 4 - Effect of genetic group on pregnancy rate, calving rate and weaning rate in 'one year' system

\begin{tabular}{lccc}
\hline & $\begin{array}{c}\text { Prenhez } \\
\text { Pregnancy } \\
\%\end{array}$ & $\begin{array}{c}\text { Parição } \\
\text { Calving } \\
\%\end{array}$ & $\begin{array}{c}\text { Desmama } \\
\text { Weaning } \\
\%\end{array}$ \\
\hline $\begin{array}{l}\text { Hereford } \\
\text { Hereford }\end{array}$ & $63,31^{\mathrm{A}}$ & $58,27^{\mathrm{A}}$ & $51,80^{\mathrm{A}}$ \\
3/4 Hereford-1/4 Nelore $(1 / 4 \mathrm{~N})$ & $69,18^{\mathrm{A}}$ & $58,90^{\mathrm{A}}$ & $48,63^{\mathrm{A}}$ \\
$3 / 4$ Hereford-1/4 Nellore/ $(1 / 4 \mathrm{~N})$ & $41,82^{\mathrm{B} * *}$ & $33,64^{\mathrm{B}^{* *}}$ & $26,36^{\mathrm{B}^{* *}}$ \\
5/8 Hereford-3/8 Nelore(3/8 N) & & & \\
$5 / 8$ Hereford $-3 / 8$ Nellore $(3 / 8 \mathrm{~N})$ & & & 2 \\
\hline
\end{tabular}

${ }^{* *} \mathrm{P}<0,01\left(^{* *} P<.01\right)$. 
concepção foram mais afetadas pelo peso vivo, condição corporal ou taxa de mudança do peso vivo em raças e cruzas com maior tamanho corporal, mais tardias que raças britânicas de maturação mais precoce. Com condições estacionais que podem comprometer a nutrição disponível para primíparas, a fertilidade de raças com maturação tardia está sob maior risco.

Na Tabela 5 observa-se um estudo em retrospectiva das variáveis peso, ganho de peso, condição corporal e idade das novilhas no 'sistema um ano' e respectivos erros padrão, de acordo com o resultado do diagnóstico de gestação por palpação trans-retal.

As novilhas que não conceberam foram, desde a desmama, até o final do acasalamento, significativamente mais leves $(\mathrm{P}<0,01)$. As diferenças de peso à desmama aumentaram até o final do acasalamento. A diferença de peso entre prenhas e não gestantes foi de 15,18 e $21 \mathrm{~kg}$ na desmama, final dos tratamentos pós-desmama (28/08) e início do acasalamento, respectivamente. Fêmeas mais leves à desmama deveriam ter recebido tratamento nutricional diferenciado para que aumentasse sua probabilidade de prenhez, conforme já demonstrado por Varner et al. (1977), Pereira Neto \& Lobato (1998) e Beretta \& Lobato (1998). A importância do peso à desmama no 'sistema um ano' novamente tornou-se evidente neste trabalho, concordando com Patterson et al. (1992) e Buskirk et al. (1995).

A CC das novilhas não gestantes foi inferior a CC das novilhas prenhas $(\mathrm{P}<0,01)$ desde a desmama até o final do acasalamento. Na desmama, a CC das novilhas que não viriam a conceber foi 0,3 pontos porcentuais inferior a $\mathrm{CC}$ das novilhas que seriam futuramente diagnosticadas como prenhas. No final do acasalamento essa diferença diminuiu para 0,2 . A $\mathrm{CC}$ é um dos indicadores do estado nutricional que mais diretamente se associa com percentagem de prenhez (Rice, 1991).

Novilhas prenhas realizaram maior $\operatorname{GDM}(\mathrm{P}<0,01)$ quando comparadas ao GDM das não gestantes desde o período nascimento-desmama até o início do acasalamento. De acordo com Topps (1977), McShane et al. (1989) e Hall et al. (1995), planos baixos de nutrição retardam o acasalamento de novilhas com um ano de idade

Novilhas diagnosticadas prenhas foram, em média, seis dias mais velhas $(\mathrm{P}<0,05)$ no início do acasalamento do que as novilhas que não conceberam. Este dado está de acordo com Milagres et al. (1979)
Tabela 5 - Médias estimadas ( \pm erro-padrão) de peso, ganho de peso, condição corporal e idade das novilhas diagnosticadas como prenhas e não gestantes no 'sistema um ano'

Table 5 - Least square means( \pm s.e.) of live weight, average daily gain, body condition and age of heifers pregnants and non-pregnants in 'one year' system

\begin{tabular}{lcc}
\hline Item & Prenha & Não gestante \\
& Pregnant & Non-pregnant \\
\hline
\end{tabular}

Peso, kg

Live weight, $\mathrm{kg}$

Nascimento

Birth

Desmama

Weaning

02/06/1994

$30 / 06 / 1994$

$27 / 07 / 1994$

$28 / 08 / 1994$

$06 / 10 / 1994$

$10 / 11 / 1994$

25/01/1995

$31,34 \pm 0,3$

$31,81 \pm 0,3$

Condição corporal

Bodycondition

Desmama

Weaning

02/06/1994

$30 / 06 / 1994$

27/07/1994

28/08/1994

$06 / 10 / 1994$

$10 / 11 / 1994$

25/01/1995

$161,04 \pm 1,4$

$146,73 \pm 1,8 * *$

$161,61 \pm 1,4$

$169,77 \pm 1,4$

$184,35 \pm 1,5$

$198,34 \pm 1,6$

$216,54 \pm 1,6$

$248,36 \pm 1,8$

$287,32 \pm 1,8$

$146,58 \pm 1,7 * *$

$153,53 \pm 1,7 * *$

$167,26 \pm 1,8 * *$

$180,88 \pm 2,0 * *$

$196,23 \pm 1,9 * *$

$227,24 \pm 2,2 * *$

$267,97 \pm 2,2 * *$

Idade acasalamento

(dias)

Age at mating (days)

GDM, kg/d

Average daily gain, $\mathrm{kg} / \mathrm{d}$

Nascimento-desmama $\quad 0,677 \pm 0,00$

Birth-Weaning

Sistemas alimentares $\quad 0,422 \pm 0,00$

(02/6-28/08)

Feeding system

(06/02-08/28)

Nascimento-

acasalamento

$$
3,6 \pm 0,03 \quad 3,3 \pm 0,03 * *
$$

$3,2 \pm 0,02$

$3,0 \pm 0,02$

$3,0 \pm 0,02$
$3,1 \pm 1,50$

$2,9 \pm 0,03 * *$

$3,3 \pm 0,02$

$3,5 \pm 0,01$

$3,6 \pm 0,01$

$3,8 \pm 0,02$

$2,8 \pm 0,02 * *$

$3,0 \pm 1,80 * *$

$3,1 \pm 0,02 * *$

$3,3 \pm 0,02 * *$

$3,4 \pm 0,02 * *$

$413,5 \pm 1,1$

$3,6 \pm 0,02 * *$

$407,8 \pm 1,4^{*}$

Birth-Mating

Início-final

acasalamento

Beginning-end mating

${ }^{* *} P<0,01$ (** $\left.P<.01\right)$.

* $\mathrm{P}<0,05$ ( $\left.{ }^{*} P<.05\right)$.

e Bergmann \& Hohenboken (1992), os quais constataram taxas mais altas de concepção em novilhas mais velhas no início da estação de acasalamento. 


\section{Conclusões}

Os índices reprodutivos desse estudo, observados no acasalamento aos 14-15 meses foram semelhantes aos índices médios do rebanho de corte do Rio Grande do Sul com acasalamento aos 36 meses.

Sistemas alimentares (pastagem, campo suplementado e silagem) no outono-inverno pós-desmama determinaram desempenho reprodutivo similar em novilhas de corte no 'sistema um ano', sendo a escolha por uma dessas técnicas a identificação da relação entre preço produto/preço insumos, determinando sua eficiência econômica.

O grau de sangue zebuíno em novilhas no 'sistema um ano' foi decisivo no desempenho reprodutivo. Novilhas Hereford e 1/4 Nelore-Hereford apresentaram maior eficiência reprodutiva quando primíparas aos 24 meses que novilhas 3/8 Nelore-Hereford

As novilhas prenhes no acasalamento com um ano de idade foram mais pesadas, mais velhas, tiveram melhor condição corporal e maiores ganhos médios diários desde o seu nascimento até o final do primeiro acasalamento que as não gestantes.

\section{Agradecimento}

Ao Méd.Vet. Valter José Potter, proprietário da Empresa Agropecuária Guatambu, pelo financiamento do trabalho e pela cessão das instalações.

Ao Médico Veterinário Adriano Garcia Rosado Jr. e à Zootecnista Luciana Pötter, pelo auxílio na coleta dos dados experimentais.

\section{Literatura Citada}

BAILEY, C.M.; HANKS, D.R.; FOOTE, W.D. et al. Maternal characteristics of young dams representing Bos taurus and Bos indicus x Bos taurus breed types. Journal of Animal Science, v.66, n.5, p.1144-1152, 1988.

BERGMANN, J.A.G.; HOHENBOKEN, W.D. Prediction of fertility from calfhood traits of Angus and Simenthal heifers. Journal of Animal Science, v.70, n.8, p.2611-2621, 1992.

BUSKIRK, D.D.; FAULKNER, D.B.; IRELAND, F.A. Increased postweaning gain of beef heifers enhances fertility and milk production. Journal of Animal Science, v.73, n.4, p.937946, 1995.

CACHAPUZ, J.M.S. O panorama setorial da bovinocultura de corte gaúcha no processo de integração do MERCOSUL. 2.ed. Porto Alegre: EMATER, 1995. 68p. (Série Realidade Rural, 7).

CARTER, A.H.; COX, E.H. Observations on yearling mating of beef cattle. In: NEW ZEALAND SOCIETY OF ANIMAL PRODUCTION, 33., 1973, Palmerston North. Proceedings... Palmerston North: New Zealand Society of Animal Production, 1973. p.94-113.

R. Bras. Zootec., v.31, n.3, p.1388-1395, 2002 (suplemento)
ELLIS, R.M. The relationship between percentage calving and weight at joining in yearling Hereford heifers. In: AUSTRALIAN SOCIETY OF ANIMAL PRODUCTION, 10., 1974, Sydney. Proceedings... Sydney: 1974. p.55-58.

FERREL, C.L. Effects of postweanig rate of gain on onset of puberty and productive performance of heifers of different breeds. Journal of Animal Science, v.55, n.6, p.12721284, 1982.

GREGORY, K.E.; LASTER, D.B.; CUNDIFF, L.V. et al. Heterosis and breed maternal and transmitted effects in beef cattle. II. Growth rate and puberty in females. Journal of Animal Science, v.47, n.5, p.1042-1053, 1978.

HALL, J.B.; SHILLO, K.K.; FITZGERALD, B.P. et al. Effects of recombinant bovine somatotropin and dietary energy intake on growth, secretion of luteinizing hormone, follicular development, and onset of puberty in beef heifers. Journal of Animal Science, v.72, n.3, p.709-718, 1995.

LASTER, D.B.; SMITH, G.M.; CUNDIFF, L.V. et al. 1979. Characterization of biological types of cattle (cycle II) II. Postweaning growth and puberty of heifers. Journal of Animal Science, v.48, n.3, p.500-507, 1979.

LOWMAN, B.G.; SCOTT, N.; SOMERVILLE, S. Condition scoring beef cattle. Edinburgh: East of Scotland College of Agriculture, 1973. 8p. (Bulletin, 6).

MAGALHÃES, F.R.; PEREIRA, P.A.; LOBATO, J.F.P. Influência da idade ao primeiro parto no desempenho reprodutivo de novilhas de corte. In: REUNIÃO ANUAL DA SOCIEDADE BRASILEIRA DE ZOOTECNIA, 27., 1990, Campinas. Anais...Campinas: Sociedade Brasileira de Zootecnia, 1990. p.369.

McMILLAN, W.H.; McCALL, D.G. Are yearling heifer mating and more productive beef cows breeds a worthwhile use of winter feed ? In: NEW ZEALAND SOCIETY OF ANIMAL PRODUCTION, 51., 1991, Palmerston North. Proceedings... Palmerston North: New Zealand Society of Animal Production, 1991. p.265-269.

McMILLAN, W.H.; MORRIS, C.A.; McCALL, D.G. 1992. Modelling herd efficiency in liveweight-selected and control Angus cattle. In: NEW ZEALAND SOCIETY OF ANIMAL PRODUCTION, 52., 1992, Palmerston North. Proceedings... Palmerston North: New Zealand Society of Animal Production, 1992. p.145-147.

McSHANE, T.M.; SCHILLO, K.K.; BOLING, J.A. et al. Effects of recombinant DNA-derived somatotropin and dietary energy intake on development of beef heifers. I. Growth and puberty. Journal of Animal Science, v.67, n.8, p.22302237, 1989.

MIELITZ NETTO, C.G.A. Modernização e diferenciação na bovinocultura de corte brasileira. Campinas: Universidade Estadual de Campinas, 1994. 224p. Tese (Doutorado em Economia) - Universidade Estadual de Campinas, 1994.

MILAGRES, J.C.; DILLARD, E.V.; ROBINSON, O.W. Influences of age and early growth on reproductive performance of yearling Hereford heifers. Journal of Animal Science, v.48, n.5, p.1089-1095, 1979.

MORENO, J.A. Clima do Rio Grande do Sul. Porto Alegre: Secretaria da Agricultura, 1961. 42p.

NATIONAL RESEARCH COUNCIL - N.R.C. Nutrient requirement of beef cattle. 7.ed. Washington, D.C.: 1996. $242 p$.

NUÑEZ-DOMINGUEZ, R.; CUNDIFF, L.V.; DICKERSON, G.E. et al. Lifetime production of beef heifers calving first at two vs. three years of age. Journal of Animal Science, v.69, n.9, p.3467-3479, 1991. 
PATTERSON, D.J.; CORAH, L.R.; BRETHOUR, J.R. et al. Evaluation of reproductive traits in Bos taurus and Bos indicus crossbred heifers: relationship of age at puberty to length of the postpartum interval to estrus. Journal of Animal Science, v.70, n.5, p.1994-1999, 1992.

PATTERSON, D.J.; CORAH, L.R.; BRETHOUR, J.R. et al. Evaluation of reproductive traits in Bos taurus and Bos indicus crossbred heifers: effects of postweaning energy manipulation. Journal of Animal Science, v.69, n.6, p.23492361, 1991.

PATTERSON, D.J.; CORAH, L.R.; KIRACOFE, G.H. et al. Conception rate in Bos taurus and Bos indicus crossbred heifers after postweaning energy manipulation and synchronization of estrus with melengestrol acetate and fenprostalene. Journal of Animal Science, v. 67, n.5, p.11381142, 1987.

PEREIRA NETO, O.A.; LOBATO, J.F.P. Efeitos da ordem de utilização de pastagens nativas melhorada no desenvolvimento e comportamento reprodutivo de novilhas de corte. Revista Brasileira de Zootecnia, v.27, n.1, p.60-65, 1998.

PÖTTER, L.; LOBATO, J.F.P.; MIELITZ NETTO, C.G. Produtividade de um modelo de produção para novilhas de corte primíparas aos dois, três e quatro anos de idade. Revista Brasileira de Zootecnia, v.27, n.3, p.613-615, 1998.

REYNOLDS, W.L.; URICK, J.J.; KNAPP, B.W. et al. Maternal breed of sire effects on postweaning performance of firstcross heifers and production of 2 year-old heifers characteristics. Journal of Animal Science, v.69, n.11, p.4368-4376, 1991.

RICE, L.E. Nutrition and the development of replacement heifers. Veterinary Clinics of North America. Food Animal Practice, v.7, n.1, p.27-42, 1991.

ROCHA, M.G. Desenvolvimento e características de produção e reprodução de fêmeas de corte primíparas aos dois anos de idade. Porto Alegre: Universidade Federal do Rio Grande do Sul, 1997. 244p. Tese (Doutorado em Zootecnia) Universidade Federal do Rio Grande do Sul, 1997.

SAS INSTITUTE. Statistical analysis systems user's guide, 4.ed. Versão 6, Cary: 1989. 1042p.

SAWYER, G.D.; BARKER, D.J.; MORRIS, J. 1991a. Performance of young breeding cattle in commercial herds in the south-west of Western Australia I. Liveweight, body condition, conception and fertility in heifers. Australian Journal of Experimental Agriculture, v.31, n.4, p.431-441, 1991a.
SAWYER, G.D.; BARKER, D.J.; MORRIS, J. Performance of young breeding cattle in commercial herds in the south-west of Western Australia I. liveweight, body condition, conception and fertility in first calf heifers. Australian Journal of Experimental Agriculture, v.31, n.4, p.443-454, 1991 b.

SHORT, R.E.; BELLOWS, R.A. Relationship among weight gains, age at puberty and reproductive performance in heifers. Journal of Animal Science, v.32, n.5, p.1964-1970, 1971.

STEEL, R.G.D.; TORRIE, J.H. Bioestadística; principios y procedimentos. Mexico: McGraw-Hill, 1980. 622p

TOPPS, J.H. The relationship between reproduction and undernutrition in beef cattle. World Review of Animal Production, v.13, n.2, p.43-49, 1977.

VARNER, L.W.; BELLOWS, R.A.; CHRISTENSEN, D.S. A management system for wintering replacement heifers. Journal of Animal Science, v.44, n.2, p.165-171, 1977.

VERCOE, J.E.; FRISH, J.E. Animal breeding for improved productivity. In: NUTRITIONAL LIMITS TO ANIMAL PRODUCTION FROM PASTURES, 1981, St. Lucia. Proceedings... St. Lucia: CSIRO, 1982. p.327-342.

WERTH, L.A.; AZZAM, S.M.; NIELSEN, M.K. et al. Use of a simulation model to evaluate the influence of reproductive performance and management decisions on net income in beef production. Journal of Animal Science, v.69, n.8, p.47104721, 1991.

YOUNG, L.D.; LASTER, D.B.; CUNDIFF, L.V. et al. Characterization of biological types of cattle. IX. Postweaning growth and puberty of three-breed cross heifers. Journal of Animal Science, v.47, n.3, p.843-852, 1978. 